\title{
Precision Timing Calorimeter for High Energy Physics
}

\author{
Dustin Anderson, Artur Apresyan, Adolf Bornheim, Javier Duarte, Cristián Peña, Maria Spiropulu, Jason Trevor, Si Xie ${ }^{\mathrm{a}}$, Anatoly \\ Ronzhin $^{\mathrm{b}}$ \\ ${ }^{a}$ California Institute of Technology, Pasadena, CA 91125 \\ ${ }^{b}$ Fermi National Accelerator Laboratory PO Box 500, Batavia, IL 60510-5011 USA
}

\begin{abstract}
Scintillator based calorimeter technology is studied with the aim to achieve particle detection with a time resolution on the order of a few $10 \mathrm{ps}$ for photons and electrons at energies of a few $\mathrm{GeV}$ and above. We present results from a prototype of a 1.4x $1.4 \times 11.4$ $\mathrm{cm}^{3}$ sampling calorimeter cell consisting of tungsten absorber plates and Cerium-doped Lutetium Yttrium Orthosilicate (LYSO) crystal scintillator plates. The LYSO plates are read out with wave lengths shifting fibers which are optically coupled to fast photo detectors on both ends of the fibers. The measurements with electrons were performed at the Fermilab Test Beam Facility (FTBF) and the CERN SPS H2 test beam. In addition to the baseline setup plastic scintillation counter and a MCP-PMT were used as trigger and as a reference for a time of flight measurement (TOF). We also present measurements with a fast laser to further characterize the response of the prototype and the photo sensors. All data were recorded using a DRS4 fast samplig digitizer. These measurements are part of an R\&D program whose aim is to demonstrate the feasibility of building a large scale electromagnetic calorimeter with a time resolution on the order of 10 picoseconds, to be used in high energy physics experiments.
\end{abstract}

Keywords: Time of flight detector, Precision timing, LYSO, MCP, pico-second timing

PACS: 29.40.Mc, 29.40.Vj, 06.60.Jn

\section{Introduction}

Modern detector technologies, in particular the availability of ${ }_{29}$ fast and precise digitizing and sampling electronics, enable tim- ${ }_{30}$ ing measurements with a precision of order ps. There is a broad ${ }_{31}$ range of developments ongoing introducing detectors with such ${ }_{32}$ precision into particle physics experiments. The ability to use ${ }_{33}$ 4-dimensional information greatly enhances the event recon- ${ }_{34}$ struction capability. A common application is the improvement ${ }_{35}$ of the signal to noise ratio of a given measurement by using the ${ }_{36}$ consistency of a time of arrival measurement of a multi particle ${ }_{37}$ final state or the consistency of a location in 4D space with $\mathrm{a}_{38}$ vertex location measured with a tracking device.

The high luminosity upgrade of the Large Hadron Collider ${ }_{40}$ (HL-LHC) at CERN [1] is expected to provide instantaneous ${ }_{41}$ luminosities of $5 \times 10^{34} \mathrm{~cm}^{-2} \mathrm{~s}^{-1}$. The enhanced data rates will ${ }_{42}$ provide the datasets necessary to perform precision measure- ${ }_{43}$ ments of the Higgs couplings, probe rare Higgs processes, study ${ }_{44}$ the scattering of longitudinally polarized W bosons, and search ${ }_{45}$ for physics beyond the standard model.

The rate of simultaneous proton-proton interactions per bunch ${ }_{47}$ crossing (pileup) is projected to reach an average of 140 to $200{ }_{48}$ The large amount of pileup increases the likelihood of confu- ${ }_{49}$ sion in the reconstruction of events of interest, due to the con- ${ }_{50}$ tamination from particles produced in different pileup interactions. The ability to discriminate between jets produced in the events of interests, especially those associated with the vector ${ }_{53}$

${ }^{*}$ Corresponding author : Adolf Bornheim, bornheim@ @ep.caltech.edu

Preprint submitted to Elsevier boson fusion processes, and jets produced by pileup interactions, will be degraded, the missing transverse energy resolution will deteriorate, and several other physics objects performance metrics will suffer.

One way to mitigate pileup confusion effects, complementary to precision tracking methods, is to perform a time of arrival measurement associated with a particular layer of the calorimeter, allowing for a time assignment for both charged particles and photons. Such a measurement with a precision of about 20 to $30 \mathrm{ps}$, when unambiguously associated to the corresponding energy measurement, will significantly reduce the inclusion of pileup particles in the reconstruction of the event of interest given that the spread in collision time of pileup interactions is about 200 ps. The association of the time measurement to the energy measurement is crucial, leading to a prototype design that calls for the time and energy measurements to be performed in the same active detector element.

The detectors operated at the LHC have very high performance tracking devices. They allow to associate charged particles to primary interaction vertices with good precision, even in very dense environments such as HL-LHC. Photons however are mostly identified by the electromagnetic calorimeters. In particular jets consist on average of $30 \%$ neutral mesons which to a large fraction get detected by their decay into photons. Some key physics signatures for the physics at HL-LHC contain high energy photons, most notably Higgs boson decays into photons. It is in this context that we study the possibility of measuring the time of arrival of particles with a calorimetric device, with an emphasis on timing measurement of photons in the energy 


\section{Precision timing technologies}

Readout electronics capable of ps precision timing measurements are readily available. The challenge of building a full scale detector system for a particle physics detector lies in the primary sensing elements of the detector.

Micro channel plate detectors (MCP) have been shown to enable the detection of single ionizing particles with a precision of a few ps. They could be used as a single layer precision timing device or as an active element in a calorimeter. We have done studies demonstrating that the time structure of electromagnetic showers is sufficiently uniform so that a MCP as an active layer would allow the measurement of the time of ar- ${ }^{110}$ rival of electromagnetic particles with a precision of a few $10^{111}$ ps [2]. Equipping a large particle physics detector in a high ${ }^{112}$ particle flux environment as the HL-LHC with a MCP based ${ }^{113}$ timing detector poses various challenges which are an area of ${ }^{114}$ active research [3].

Semiconductor based sensors are being explored for preci- ${ }^{116}$ sion timing applications as well. On the single sensor level the $\mathrm{e}^{117}$ timing precision for single charged particle detection is on the $e^{118}$ order of $100 \mathrm{ps}$ [4]. It is being studied if the time of arrival of ${ }^{119}$ showers in calorimeters could be measured with significantly ${ }^{120}$ better precision utilizing the large number of synchronous par- ${ }^{121}$ ticles in a shower and showers spreading over a large number ${ }^{122}$ of independent sensors.

For the studies presented in this document we focus on mea- ${ }^{124}$ surements of the time of flight using sampling calorimeters ${ }^{125}$ based on LYSO crystals. Due to its very high light yield $\left(\sim 30 \mathrm{~K}^{126}\right.$ photons/MeV) [5], and radiation tolerance [6], LYSO is the ac- ${ }^{127}$ tive element of one of the options considered for the upgrade of the Compact Muon Solenoid (CMS) detector at the HLLHC [7].

In Figure 1 we present a simplified illustration of the major ${ }_{129}$ time scales associated to the timing measurement using a mono-130 lithic crystal calorimeter [8]. Upon entering the crystal, the ${ }_{131}$ photon or electron travels at the speed of light; these particles ${ }_{132}$ interact and beginning to shower, producing scintillation light.133 The time between the entry of the photon into the crystal and $d_{134}$ the first interaction is denoted by $t_{I}$ and for high energy imping-135 ing particles it is the shower development time. The time period ${ }_{136}$ associated with the conversion of the incident photon to scintil-137 lation light is denoted by $t_{S}$. The scintillation light travels from ${ }_{138}$ the point of interaction to the photo detector at a velocity $c / \hat{n}, 139$ where $\hat{n}$ is the effective index of refraction of the crystal. The $e_{140}$ time associated with the propagation of the scintillation light ${ }_{141}$ from the point of interaction to the photo detector is denoted by 142 $t_{P}$. Once the scintillation light reaches the photo detector, the ${ }_{143}$ photons are converted into an electrical signal. The time period ${ }_{144}$ associated with this process is known as the photo detector sig-145 nal transit time, $t_{T}$. Finally, the data acquisition (DAQ) system ${ }_{146}$ has a characteristic time constant $t_{D}$. Each of time periods de-147 scribed above will fluctuate or jitter on an event-by-event basis, 148 contributing to the total time resolution.

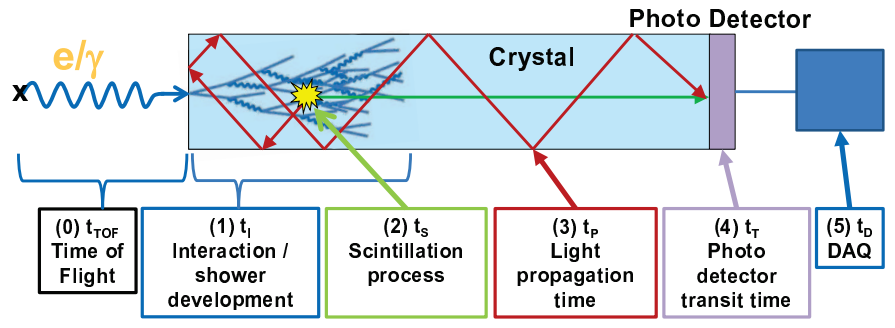

Figure 1: Schematic cartoon of the most relevant contributions of a timing measurement with a monolithic, large scintillating crystal. The incident particle impinges on the crystal face from the left. Characteristic times of various contributions are defined in the colored boxes and defined in the text.

To characterize the time resolution of an inorganic crystal scintillator calorimeter we study the contributions due to fluctuations in the shower development, scintillation process, and light propagation to the photo detector. The very large number of scintillation photons in a LYSO crystal minimizes the fluctuations associated with the creation and transit of each particular scintillation photon for a LYSO-based detector providing a robust average. This in turn, allows the study of the effect of the light propagation time. For very high energy showers the number of scintillation photons is very large, even if crystals with lower light yield are used. The CMS ECAL, using lead tungstate crystals, features a time resolution for electrons of about $150 \mathrm{ps}$ and a resolution of $70 \mathrm{ps}$ among channels belonging to the same electromagnetic shower [9]. This also demonstrates the feasibility of calibrating a large scale detector in situ and maintain its performance in a challenging radiation environment.

\section{Timing in LYSO Crystal-based Calorimeters}

Besides the effects of the photo detector and DAQ electronics, timing performance in LYSO-based calorimeters is driven by three factors: shower profile fluctuations, scintillation light production, and optical transport. Stochastic processes during the development of an electromagnetic shower affect the time at which signals are observed, as both the transverse size and the depth of the shower can fluctuate event by event. Random processes in the scintillation mechanism and the randomization of the optical paths for the scintillation light affect both the speed of the signal formation and the time jitter. We study these effects using various independent experimental setups. In particular we present here studies of a LYSO-tungsten Shashlik calorimeter cell with a size of $1.4 \times 1.4 \times 11.4 \mathrm{~cm}^{3}$, containing 29 layers of LYSO crystal plates with a thickness of $1.5 \mathrm{~mm}$, interleaved with tungsten absorber plates. The scintillation light is read out with four wave length shifting fibers of about $1 \mathrm{~mm}$ diameter. The usage of the wavelength shifter enhances the efficiency of the light extraction from the LYSO tiles, but it introduces an additional time constant in the rise time [10]. In the configuration used here DSB is used as a wavelength shifter which has a time constants of 2 to 3 ns. A matrix of 4 by 4 
such cells has been assembled and tested independently with ${ }_{187}$ electrons in the energy range between $4 \mathrm{GeV}$ and $250 \mathrm{GeV}$ in ${ }_{188}$ beam facilities in CERN and FNAL. The energy resolution of this prototype was measured to be $\frac{10 \%}{\sqrt{E}} \oplus 1 \%$ and the position resolution to be $0.6 \mathrm{~mm}$ for $100 \mathrm{GeV}$ electrons. The radiation hardness of the LYSO crystals was tested up to $80 \%$ of the fluence expected expected at HL-LHC assuming an integrated luminosity of $3 \mathrm{ab}^{-1}$. This qualifies the technology choice of a LYSO-tungsten Shashlik calorimeter as a viable option for a precision electromagnetic calorimeter. Using a single Shashlik calorimeter cell, read out with DSB1 fibers, Hamamatsu MCPs [12] and DRS4 [14] evaluation boards as DAQ system we have measured the time resolution for electron beams with energy varying between $4 \mathrm{GeV}$ and $150 \mathrm{GeV}$. A Photek MCP [13] was used as a reference time measurement for the incoming electron.

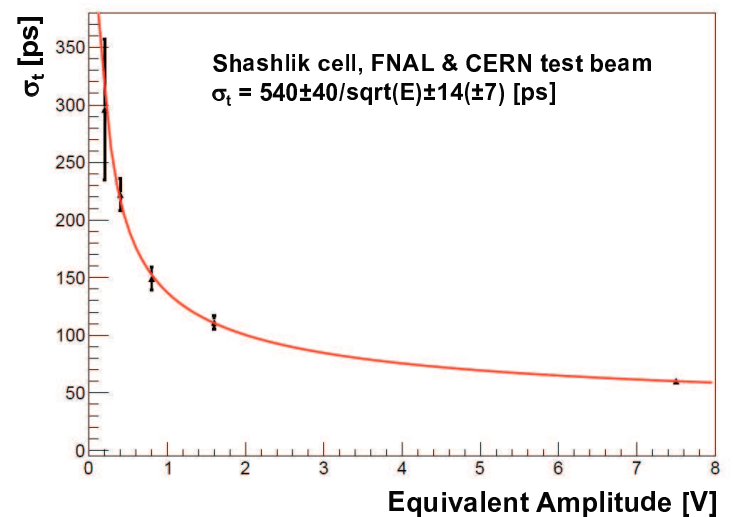

Figure 2: The time resolution measured using the LYSO-tungsten shashlik ${ }_{19}$ calorimeter with light extracted using DSB1 wave length shifting fibers is plotted as a function of the effective signal amplitude from the MCP, and fitted to $\mathrm{a}^{200}$ $1 / \sqrt{E}$ functional form.

The time resolution is plotted as a function of the beam en-203 ergy in Figure 2. Here we show the result as a function of the effective signal amplitude from the MCP. The individual points correspond to energies of $4,8,16,32$ and $150 \mathrm{GeV}$. The containment for the single cell with the trigger counter used is about ${ }_{205}$ $80 \%$ on average. We find that the dependence of the time reso-206 lution on beam energy follows a $1 / \sqrt{E}$ functional form, reach-207 ing about $100 \mathrm{ps}$ for a beam energy of $32 \mathrm{GeV}$ and around $60_{208}$ ps at $150 \mathrm{GeV}$. A fit of a $a / \sqrt{E} \oplus c$ suggest a constant term 209 of the resolution of around $15 \mathrm{ps}$, in good agreement with out 210 estimated precision of the reference time measurement as dis-211 cussed in one of our previous publications [10].

\section{Timing Performance of the Hamatsu MCPs with DRS4 ${ }^{214}$ readout}

Utilizing a laser [11] with a pulse width of less than $30 \mathrm{ps}_{217}$ and a jitter of less than $5 \mathrm{ps}$ we measured the performance of $_{218}$ the Hamamatsu MCP sensors and the associated readout with 219 the DRS4. We exposed two MCP sensors to the same laser 220 pulses and measured the differential time resolution between 221 the two sensors without any additional optical elements in the 222 light path. As shown in Figure 3 we measure this differential 223 time resolution between the two sensors of $7.2 \mathrm{ps.} \mathrm{From} \mathrm{this}$ we may infere a single channel resolution of around $5 \mathrm{ps}$.

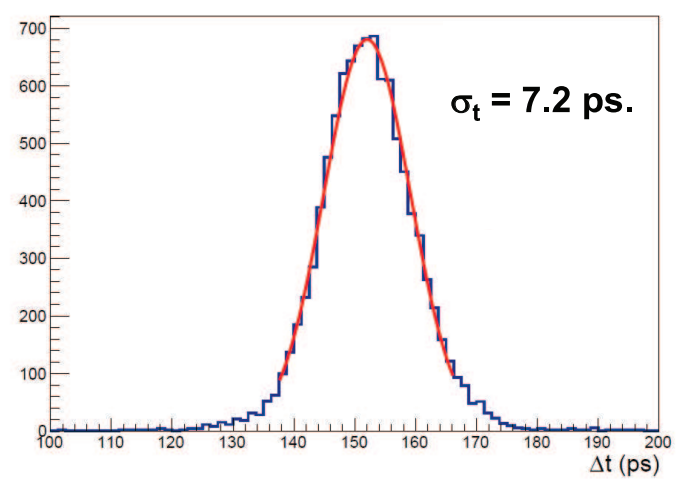

Figure 3: Differential time resolution between two Hamamatsu MCPs, measured with a laser with a pulse with of better than $30 \mathrm{ps}$. We measure the resolution to be 7.2 ps which corresponds to a single channel resolution of around 5 ps.

In previous studies $[10,2]$ we have shown the contribution of the DRS4 readout to the time resolution to be around $5 \mathrm{ps.}$ We conclude from this study that the performance of out light sensing chain for a large number of synchronous photons is indeed close the the limit of our DRS4 readout. The result of around 7 ps was achieved over a wide range of pulse intensities, covering the equivalent amplitude range of our studies with the LYSO assemblies. This results also confirms the assumption that our photo detector timing performance improves for large, synchronous pluses. Studies with a laser with significantly wider pulse width of a few ns did not yield the same good performance. This suggest that additional noise like phenomena may be induced in our setup by these very long light pulses. This is under further study and will be reported in a future communication.

\section{Advanced Pulse Reconstruction}

We have discussed previously that we do not see any evidence that the complexity of the light path in the crystal and light guiding elements has any impact on the observed pulse shape [10]. To study the impact of the observed dependency of the photo sensor and readout chain on the light pulse length we developed an advanced pulse reconstruction method. In this new method we extract the time of arrival of high energy electrons in our LYSO-tungsten Shashlik cell only from the very first part of rising edge of the measured pulse shape. Previously we used the part of the rising edge of the pulse shape in the range of $20 \%$ to $60 \%$ of the maximum amplitude of the pulse. In Figure 4 we show a comparison of our previously published results [10] and the new pulse reconstruction utilizing only the very early leading edge of the pulse from the first rise above the noise level to $30 \%$ of the maximum pulse amplitude.

We observe a significant improvement of the time resolution for high energy electrons measured with the LYSO-tungsten Shashlik cell. This suggests that the same noise like phenomena observed for long laser pulses may also affect the measurements 


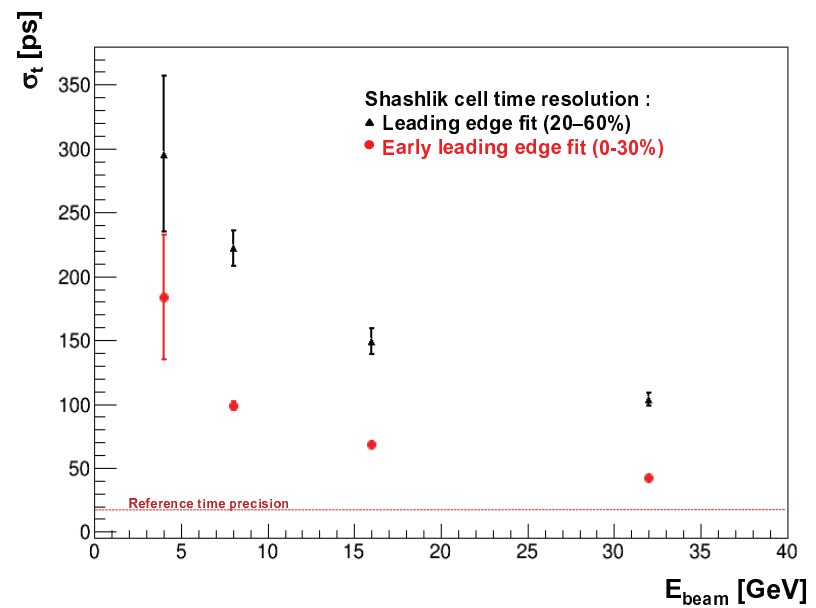

Figure 4: Comparison of the time resolution between a reference MCP and the LYSO-tungsten Shashlik cell for high energy electrons. Using only the very early part of the rising edge of the pulse we observe a significantly better timing ${ }^{273}$ performance compared to a time extraction using the entire rising edge.

using LYSO crystals. We recall that while LYSO has a very fast276 rise time its decay time constant is on the order or $40 \mathrm{~ns}$. The 277 effective rising edge of our LYSO-tungsten Shashlik cell is a 278 convolution of the scintillation rise time, the light propagation 279 time characteristics, the time characteristics of the wavelength280 shifter and the time characteristics of the photo sensor and the281 readout chain. For our LYSO-tungsten Shashlik cell we mea-282 sure a rise time of $2.1 \mathrm{~ns}$ which is close to the time constant of the DSB wave length shifter used in the setup. This dominates the rise time and stretches it to a duration for which we started to observed noise like phenomena with laser pulses of equiva-284 lent length. We conclude from this that the performance of our ${ }^{285}$ setup is currently limited by these noise like phenomena. The ${ }_{287}^{286}$ leading edge fit extracts the time of arrival information in the 28 LYSO-tungsten Shashlik cell from the early portion of the pulse $\mathrm{e}^{289}$ which is less affected by this induced noise. Further studies are ${ }_{291}^{290}$ ongoing to quantify and possibly mitigate this effect. This $i_{292}$ expected to yield a further improvement of performance.

\section{Summary}

In this study we present further characterization of the tim-299 ing performance of LYSO-based calorimeters. We had previ ${ }^{300}$ ously shown results using a $(1.7 \mathrm{~cm})^{3}$ LYSO crystal that sam- ${ }_{302}^{301}$ ples the electromagnetic showers created by electrons of vari-303 ous energies ranging from $4 \mathrm{GeV}$ to $32 \mathrm{GeV}$ at about $4.5 X_{0}{ }^{304}$ we inferred that the contribution to the time resolution from $_{306}^{305}$ event-by-event fluctuations of the shower profile, the scintilla- ${ }_{306}$ tion process, and the optical transit was less than $30 \mathrm{ps.} \mathrm{We} 308$ further study the effect of optical transit through WLS fibers ${ }^{309}$ and the light extraction efficiency in a LYSO-tungsten Shashlik ${ }_{311}^{310}$ calorimeter demonstrated that the use of a WLS fiber domi- ${ }_{312}$ nates the signal rise time of our setup which has a major impact313 on the time resolution. Studies with a fast laser suggest that ${ }^{314}$ our photo sensor and readout assembly has a differential time ${ }_{316}^{315}$ resolution of around $7 \mathrm{ps}$ while this performance degrades for for $_{317}$ long light pulses. Studies of different pulse extraction methods ${ }^{318}$ suggest that similar effects are present in our LYSO-tungsten setup. Restricting the time measurement to the very early part of the pulse yields a significant improvement of the timing performance. The best value achieved around $40 \mathrm{ps}$ for a $32 \mathrm{GeV}$ electron beam, compared to the previously achieved $100 \mathrm{ps}$.

In summary we demonstrated that a time resolution of a few 10 ps can be achieved with a LYSO based calorimeter with a design suitable for a high precision electromagnetic calorimeter to be operated at HL-LHC.

\section{Acknowledgments}

We would like to thank Erik Ramberg, Sergey Los and Aria Soha for their help and support and the FNAL and CERN TBF for the beam delivery and control. We thank Randy Ruchti for providing us with DSB fibers and Eileen Hahn for polishing the fibers. We would also like to thank Ewa Skup, Geoff Savage and Todd Nobel for help with the beam instrumentation, organizing and providing the supporting equipment at FTBF as well as David Bailleux and Tatiana Medvedeva for help at CERN. This work is supported by funding from Fermi Research Alliance, LLC under Contract no. DE-AC02-07CH11359 with the United States Department of Energy and from California Institute of Technology High Energy Physics under Contract DE-SC0011925 with the United States Department of Energy.

\section{References}

[1] L. Rossi, and O. Bruning,Tech. Rep. CERN-ATS-2012-236, CERN, Geneva, Aug 2012.

[2] A. Ronzhin, S. Los, E. Ramberg, et al., "Development of a new fast shower maximum detector based on microchannel plates photomultipliers (MCP-PMT) as an active element”, NIM A 759 (2014) 65

[3] http://psec.uchicago.edu/

[4] N. Cartiglia, et al., "Design optimization of ultra-fast silicon detectors", NIM A (2015), http://dx.doi.org/10.1016/j.nima.2015.04.025i

[5] L. Zhang, R. Mao, F. Yang, and R. Zhu, LSO/LYSO Crystals for Calorimeters in Future HEP Experiments, IEEE Transactions on Nuclear Science, vol. 61, pp. 483488, Feb 2014.

[6] G. Dissertori, D. Luckey, Nessi-Tedaldi, et al., Results on damage induced by high-energy protons in LYSO calorimeter crystals, NIM A 745 (2014) $1-6$.

[7] D. Contardo and J. Spalding, Tech. Rep. CERNRRB- 2013-124, CERN, Geneva, Oct 2013.

[8] A. Bornheim, et al., "Calorimeters for Precision Timing Measurements in High Energy Physics", Proceedings of CALOR2014, Journal of Physics: Conference Series 587 (2015) 012057, doi:10.1088/1742$6596 / 587 / 1 / 012057$

[9] A. Bornheim, on behalf of the CMS Collaboration, "Timing performance of the CMS electromagnetic calorimeter and prospects for the future ", TIPP2014,

[10] A. Bornheim, et al., "On timing properties of LYSO-based calorimeters", NIM A 794 (2015) 7-14.

[11] PiLas, Advanced Laser Diode Systems, www.alsgmbh.com

[12] http://www.hamamatsu.com/resources/pdf/etd/R3809U50_TPMH1067E09

[13] http://www.photek.com/pdf/datasheets/detectors/DS006_Photomultipliers.pdf

[14] S. Ritt, R. Dinapoli and U. Hartmann, "Application of the DRS chip for fast waveform digitizing ", NIM A 623 (2010) 486-488.

[15] M. Albrecht, K. Andert, P. Anselmino, et al., Scintillators and Wavelength Shifters for the Detection of Ionizing Radiation, Proceedings of the 8th Conference on astroparticle, particle and space physics, detectors and medical physics applications, pp. 502511, 2003. 